\title{
A Parallel Data Distribution Management Algorithm
}

\author{
Moreno Marzolla, Gabriele D'Angelo \\ Dept. of Computer Science and Engineering \\ University of Bologna, Italy \\ Email:moreno.marzolla@unibo.it,.g.dangelo@unibo.it
}

\author{
Marco Mandrioli \\ Email:m.mandrioli@live.it
}

Abstract-Identifying intersections among a set of $d$ dimensional rectangular regions ( $d$-rectangles) is a common problem in many simulation and modeling applications. Since algorithms for computing intersections over a large number of regions can be computationally demanding, an obvious solution is to take advantage of the multiprocessing capabilities of modern multicore processors. Unfortunately, many solutions employed for the Data Distribution Management service of the High Level Architecture are either inefficient, or can only partially be parallelized. In this paper we propose the Interval Tree Matching (ITM) algorithm for computing intersections among $d$-rectangles. ITM is based on a simple Interval Tree data structure, and exhibits an embarrassingly parallel structure. We implement the ITM algorithm, and compare its sequential performance with two widely used solutions (brute force and sort-based matching). We also analyze the scalability of ITM on shared-memory multicore processors. The results show that the sequential implementation of ITM is competitive with sort-based matching; moreover, the parallel implementation provides good speedup on multicore processors.

Keywords-Data Distribution Management; High Level Architecture; Parallel Algorithms; Interval Tree

\section{INTRODUCTION}

The High Level Architecture (HLA) specification [1] defines several Data Distribution Management (DDM) services to forward events generated on update regions to a set of subscription regions. For example, consider a simulation of vehicles moving on a two-dimensional terrain. Each vehicle may be interested in events happening inside its area of interest (e.g., its field of view) that might be approximated with a rectangular region centered at the vehicle position. This kind of problem also arises in the context of Massively Multiplayer Online Games, where the game engine must send updates only to players that might be affected by game events, in order to reduce computation cost and network traffic. In this paper we assume that a region corresponds to a single extent in DDM terminology), that is, a $d$-dimensional rectangle ( $d$-rectangle) in a $d$-dimensional routing space.

Spatial data structures that can solve the region intersection problem have been developed over the years; examples

${ }^{0}$ The publisher version of this paper is available at http://dx.doi.org/10.1109/DS-RT.2013.23 Please cite as: Moreno Marzolla, Gabriele D'Angelo, Marco Mandrioli. A Parallel Data Distribution Management Algorithm. Proceedings of the 2013 IEEE/ACM 17th International Symposium on Distributed Simulation and Real Time Applications (DS-RT 2013). ISBN, 978-0-7695-5138-8. include the $k$ - $d$ tree [2] and R-tree [3]. However, it turns out that simpler, less efficient solutions are actually preferred in practice and widely deployed in DDM implementations. The reason is that efficient spatial data structures tend to be complex to implement, and therefore their theoretical performance is affected by high constant factors.

The increasingly large size of computer simulations employing DDM techniques is posing a challenge to the existing solutions. As the number of regions increases, so does the execution time of the DDM service. Given the current trend in microprocessor design where a single CPU contains multiple independent execution units, significant improvements could be achieved if the existing DDM matching algorithms were capable of taking advantage of the computational power provided by multi-core processors.

There are two opportunities for parallelizing DDM algorithms. The first is based on the observation that the problem of identifying whether two $d$-rectangles intersect can be reduced to $d$ independent intersection problems among onedimensional segments (details will be given in Section III). Therefore, given an algorithm that can identify intersections among two sets of segments, we can execute $d$ instances in parallel, each computing the intersections among the projections of the extents along each dimension. The extent intersections can be easily computed from the segments overlap information.

The idea above can be regarded as the "low hanging fruit" which is easy to get, but does not solve the problem in the long run. In fact, the number of cores in modern processors is often larger than the number of dimensions of most routing spaces; this gap is likely to increase (e.g., the Tilera TILE-Gx8072 processor [4] offers 72 generalpurpose cores on the same chip, connected through an onchip mesh network). Here comes the second parallelization opportunity: distribute the regions to the available cores so that each core can work on a smaller problem. This is quite difficult to achieve on the existing DDM algorithms, since they are either inefficient (and therefore there is little incentive in splitting the workload), or inherently sequential (and therefore there is no easy way to achieve parallelism over the set of extents).

In this paper we describe the Interval Tree Matching (ITM) algorithm for solving the one-dimensional segment 
intersection problem. The algorithm uses a simple implementation of the Interval Tree data structure based on an augmented balanced search tree. Experimental performance measures indicate that the sequential version of ITM is competitive in the sequential case with the best algorithm used for DDM, namely sort-based matching. We also observed good scalability of the parallel implementation of ITM on shared-memory architectures. An important feature of ITM is that it can be used to efficiently update overlap information in a dynamic setting, that is, in case extents can be moved or resized dynamically.

This paper is organized as follows. In Section IIwe briefly review the state of the art and compare ITM with existing solutions to the DDM matching problem. In Section III we describe three commonly used algorithms for DDM: brute force, grid-based and sort-based matching. In Section IV we describe ITM and analyze its computational cost. In Section $\mathrm{V}$ we experimentally evaluate the performance of the sequential version of ITM compared with brute force and sort-based matching; additionally, we study the scalability of a parallel implementation of ITM on a multicore processor. Finally, conclusions and future works will be discussed in Section VI

\section{RELATED WORK}

DDM matching can be considered as an instance of the more general problem of identifying intersecting pairs of (hyper-)rectangles in a multidimensional metric space. Well known space-partitioning data structures such as $k$-d trees [2] and R-trees [3] can be used to efficiently store volumetric objects and identify intersections with a query object. However, spatial data structures are quite complex to implement and, although asymptotically efficient, they can be slower than less efficient but simpler solutions in many real-world situations [5]. In [6] the authors describe a rectangle-intersection algorithm in two-dimensional space that uses only simple data structures (arrays), and can enumerate all $k$ intersections among $n$ rectangles $O(n \log n+k)$ time and $O(n)$ space.

The usage of Interval Trees for DDM was first proposed in [5] where the authors used a different and more complex data structure than the one proposed here (see Section IV]. In their case, the performance evaluation on very small instances shows mixed results.

Sort-Based Matching (SBM) [7] is a widely used algorithm for enumerating all intersections among subscription and update extents, with particular emphasis on distributed simulation applications based on the High Level Architecture (HLA) specification. SBM first sorts the endpoints, and then scans the sorted set (details will be given in Section [I-D]. SBM is extended in [8] to work efficiently on a dynamic scenario where extents can be moved or resized dynamically.
Despite its simplicity and efficiency, SBM has the drawback that its sequential scan step is intrinsically serial and can not be easily parallelized. This can be a serious limitation when dealing with a large number of extents on multicore processors.

In [9] the authors propose a binary partition-based matching algorithm that has good performances in some settings, but suffers from a worst case cost of $O\left(N^{2} \log N\right)$ where $N$ is the total number of subscription and update regions. Moreover, the extension of this algorithm to the dynamic scenario seems impractical.

Layer et al. [10] describe the Binary Interval Search (BITS) algorithm. BITS can be used to efficiently count the number of intersections between two sets $A$ and $B$ of intervals in time $O((|A|+|B|) \log |B|)$. To do so, BITS performs a preprocessing phase in which two sorted arrays $B_{S}$ and $B_{E}$ are created in time $O(|B| \log |B|)$. $B_{S}$ contains the starting points of all intervals in $B$, while $B_{E}$ contains the ending points. The number of intervals in $B$ that intersect a given query interval $q=[q$. low, q.high $]$ can be computed by subtracting from $|B|$ the number of intervals which do not intersect $q$. BITS uses two binary searches in $B_{S}$ and $B_{E}$ to compute the number of intervals in $B$ whose ending point precedes q.low, and those whose starting point follows q.high. While BITS can be easily parallelized by executing the binary searches in parallel, it must be observed that the problem of enumerating all intersections can not be easily handled by BITS without substantial modifications which significantly increase its computational cost.

\section{DDM MATCHING Algorithms}

In this section we define the DDM problem and describe three well known solution algorithms that have been thoroughly investigated.

Let $\mathbf{S}=\left\{S_{1}, \ldots, S_{n}\right\}$ and $\mathbf{U}=\left\{U_{1}, \ldots, U_{m}\right\}$ be two sets of rectangular regions in $d$-dimensional space $(d$ rectangles, also called extents). $\mathbf{S}$ is the set of subscription extents, while $\mathbf{U}$ is the set of update extents. Each extent $T$ has an integer attribute $T$.id representing its index in the set it belongs to, e.g., $S_{i} . i d=i$ and $U_{j} . i d=j$. The goal of a DDM matching algorithm is to identify all intersections between a subscription and an update extent, that is, enumerating the content of the subset of $\mathbf{S} \times \mathbf{U}$ defined as

$$
\left\{S_{i} \in \mathbf{S}, U_{j} \in \mathbf{U} \mid S_{i} \cap U_{j} \neq \emptyset\right\}
$$

Figure 11 shows an example in $d=2$ dimensions with three subscription extents $\left\{S_{1}, S_{2}, S_{3}\right\}$ and two update extents $\left\{U_{1}, U_{2}\right\}$. We observe that $U_{1}$ overlaps with $S_{1}$ and $S_{3}$, while $U_{2}$ overlaps with $S_{2}$ and $S_{3}$.

The preferred way of storing the intersections uses a $n \times m$ binary matrix $\mathbf{M}$, where each element $M_{i j}=1$ if and only 


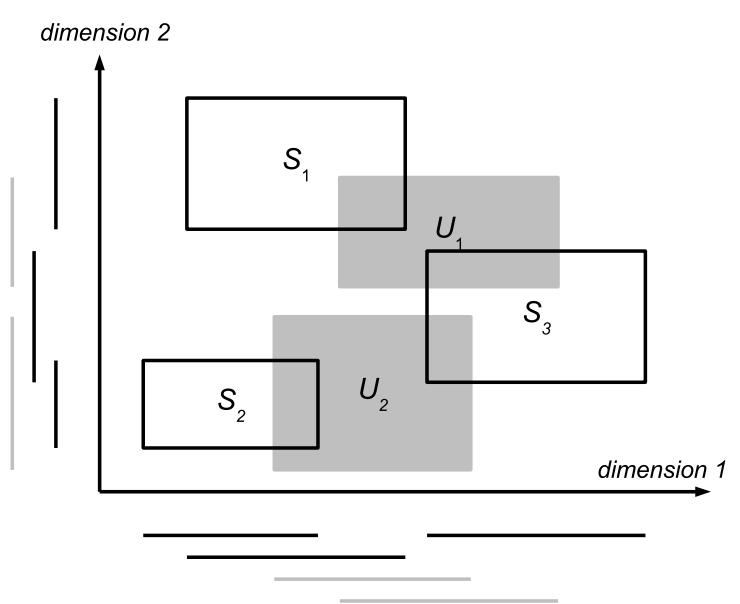

Figure 1. Data Distribution Management example in $d=2$ dimensions

\begin{tabular}{|c|}
\hline Algorithm 1 Segment intersection test \\
\hline $\begin{array}{l}\text { function INTERSECT-1D }(x, y) \\
\quad \text { return } x \text {.low }<y \text {.high } \wedge \text { y.low }<x \text {.high }\end{array}$ \\
\hline
\end{tabular}

if $S_{i}$ intersects $U_{j}$. In the case of Figure 1 we have

$$
\mathbf{M}=\left(\begin{array}{ll}
1 & 0 \\
0 & 1 \\
1 & 1
\end{array}\right)
$$

Since the number of intersections is generally much smaller than $n \times m$, matrix $\mathbf{M}$ tends to be sparse and can be stored in compressed form to reduce the memory requirement.

It is important to observe that any DDM algorithm that enumerates all $K$ intersections requires time at least $\Omega(K)$; we say that the time complexity of DDM matching algorithms is output-sensitive, since it depends on the size of the output as well as on the size of the input. Since $K \leq n m$, in the worst case we have that any algorithm has a worstcase complexity of $O(n m)$. Every algorithm that stores the result into an uncompressed intersection matrix requires time $O(n m)$ to initialize the matrix, regardless of the number of intersections. Despite this, it makes sense to try to improve the efficiency of overlap identification, since in practice this is the slower step of the DDM problem.

\section{A. Testing Intersection}

Testing whether two $d$-rectangles intersect is a key operation. When $d=1$ the problem is reduced to testing whether two segments $x=[x . l o w, x . h i g h], y=[y$. low, y.high $]$ intersect, that can be done in time $O(1)$ using Algorithm 1

For the general case $d>1$ we observe that two $d$ dimensional extents $S_{i}$ and $U_{j}$ intersect if and only if all their projections along each dimension intersect. Looking again at Figure 1, we see that the projections of $S_{1}$ and $U_{2}$ intersect along dimension 1 but not along dimension 2; therefore, $S_{1}$ and $U_{2}$ cannot intersect. On the other hand, the
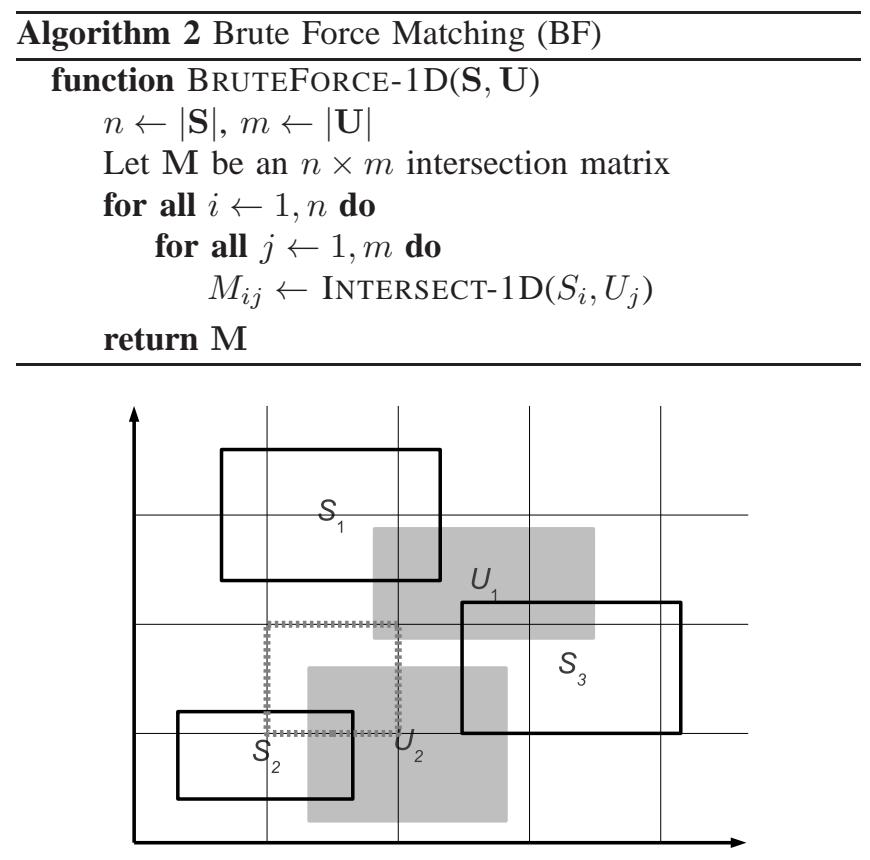

Figure 2. Grid-based DDM in $d=2$ dimensions.

projections of $S_{2}$ and $U_{2}$ intersect along both dimensions, and in fact these rectangular regions intersect in the plane.

Since dealing with segments is easier than dealing with $d$ rectangles, it is common practice in the DDM research community to define efficient algorithms for the one-dimensional case, and use them to solve the general higher dimensional case. According to the discussion above, an algorithm that enumerates all intersections among two sets of $n$ and $m$ segments in time $O(f(n, m))$ can be immediately extended to an $O(d \times f(n, m))$ algorithm for $d$-rectangles. For this reason in the rest of this paper we will consider the case $d=1$ only.

\section{B. Region-Based Matching}

The most direct approach for solving the segment intersection problem is Region-Based matching, also called Brute Force (BF) approach shown in Algorithm 2 The BF algorithm tests all $n \times m$ subscription-update pairs, and records intersection information in matrix $\mathbf{M}$.

The $\mathrm{BF}$ algorithm requires time $O(\mathrm{~nm})$, and is therefore not very efficient; despite this, it is appealing due to its simplicity. Furthermore, BF can be trivially parallelized since all iterations are independent (it is an example of embarrassingly parallel computation). When $p$ processors are available, the amount of work performed by each processor is $O(n m / p)$.

\section{Grid-Based Matching}

The Grid Based (GB) matching algorithm proposed by Boukerche and Dzermajko [11] is an improved solution to the $d$-rectangle intersection problem. It works by partitioning 
the routing space into a grid of $d$-dimensional cells. Each extent is mapped to the grid cells it overlaps with. The events produced by an update extent $U_{j}$ are sent to all subscriptions that share at least one cell in common with $U_{j}$.

The GB approach is more scalable than BF; furthermore, its performance can be tuned by choosing a suitable cell size. Unfortunately, it has some drawbacks: GB matching may report spurious overlaps, that is, may deliver events to subscribers which should not receive them. This situation is illustrated in Figure 2, the extent $U_{1}$ and $S_{2}$ share the dashed cell but do not overlap; therefore, $S_{2}$ will receive spurious notifications from $U_{1}$ that will need to be filtered out at the receiving side.

The problem of spurious events can be mitigated by applying the brute force algorithm to each grid cell. If the routing space is partitioned into $G$ cells and all extents are evenly distributed over the grid, each cell will have $n / G$ subscription extents and $m / G$ update extents. Therefore, the brute force approach applied to each cell requires $O\left(n m / G^{2}\right)$ operations; since there are $G$ cells, the overall complexity becomes $O(\mathrm{~nm} / \mathrm{G})$. In conclusion, in the ideal case the GB matching can reduce the workload by a factor $G$ with respect to BF. Unfortunately, when cells are small (and therefore $G$ is large) each extent is mapped to a larger number of cells, which increases the computation time.

\section{Sort-Based Matching}

The Sort-Based Matching algorithm proposed by Raczy et al. [7] is a simple and very efficient solution to the DDM matching problem.

In its basic version, SBM is illustrated in Algorithm 3 Given a set $\mathbf{S}$ of $n$ subscription intervals, and a set $\mathbf{U}$ of $m$ update intervals, the algorithm sorts the endpoints in nondecreasing order in the array $L$. Then, the algorithm performs a scan of the sorted vector; two sets SubscriptionSet and UpdateSet are used to keep track of the active subscription and update intervals at every point $p$. Each time the upper bound of an interval $T$ is encountered, the intersection matrix $\mathbf{M}$ is updated appropriately, depending on whether $T$ is a subscription or update extent.

As can be seen, SBM uses only simple data structures. If we ignore the time needed to initialize the matrix $\mathbf{M}$, Algorithm 3 requires time $O((n+m) \log (n+m))$ to sort the vector $L$, then time $O(n+m)$ to scan the sorted vector. During the scan phase, total time $O(n m)$ is spent to transfer the information from the sets SubscriptionSet and UpdateSet to the intersection matrix $\mathbf{M}$, assuming that the sets above are implemented as bitmaps [7]. The overall computational cost is $O((n+m) \log (n+m)+n m)$, and therefore asymptotically not better than $\mathrm{BF}$; however, the term $O(\mathrm{~nm})$ comes from simple operations on bitmaps, hence SBM is very efficient in practice [7].

While SBM is very fast, it has the drawback of not being easily parallelizable. In fact, while parallel algorithms

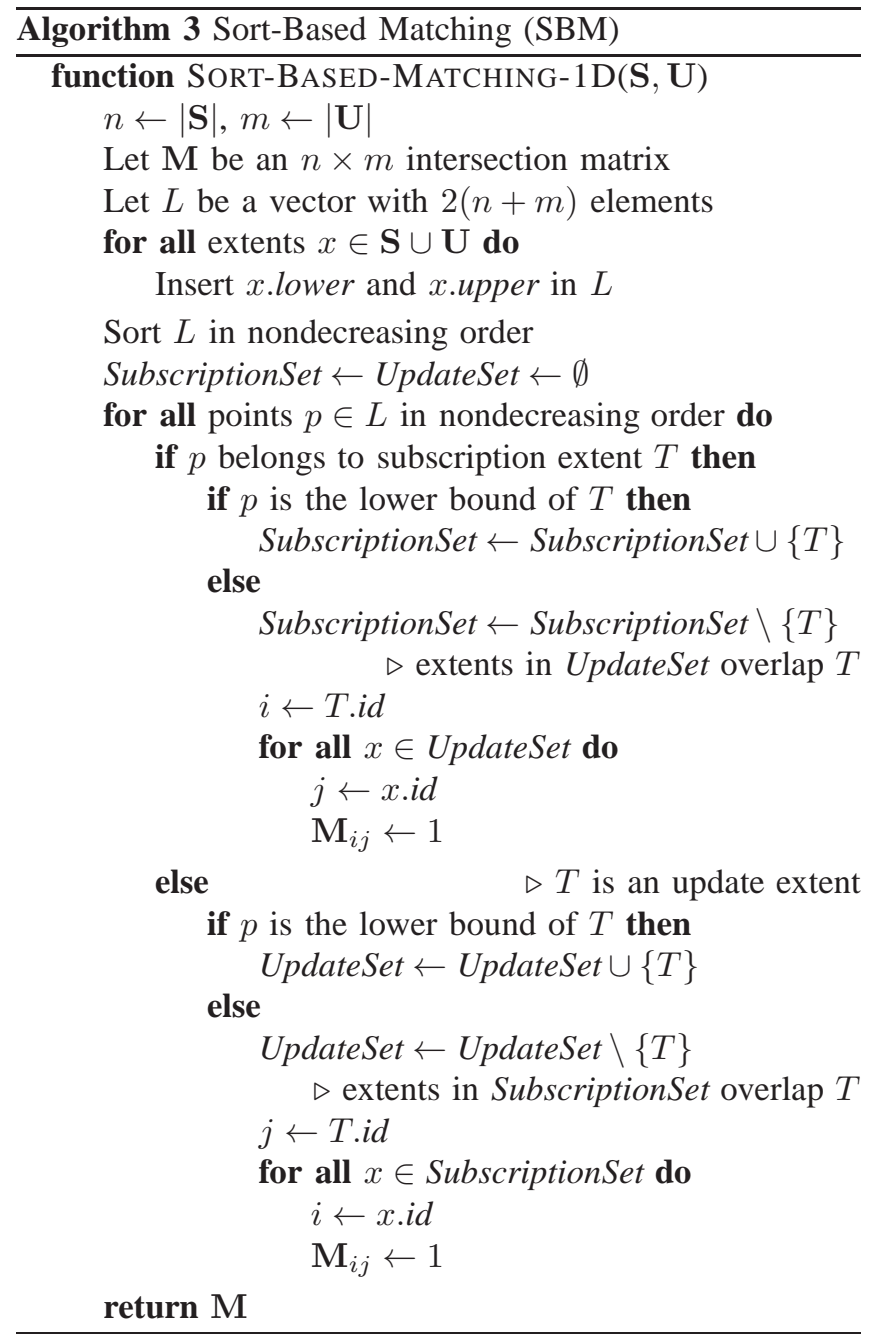

for sorting the array $L$ are known [12], the scan step is affected by loop-carried dependencies, since the content of SubscriptionSet and UpdateSet depend on their values at the previous iteration. This dependency can not be easily removed. Given the widespread availability of multi- and many-core processors, this limitation can not be ignored.

In the next section we introduce the Interval Tree Matching algorithm for computing intersections among two sets of intervals. ITM uses an augmented AVL tree data structure to store the intervals. The performance of ITM depends on the number of intersections; however we will show that ITM is faster than SBM in the scenarios considered in the literature. Furthermore, ITM can be trivially parallelized, hence further performance improvements can be obtained on shared-memory multi-core processors.

\section{INTERVAL TREE MATCHING}

ITM is a DDM matching algorithm for one dimensional segments based on the Interval Tree data structure. An Interval Tree stores a dynamic set of $n$ intervals, and 


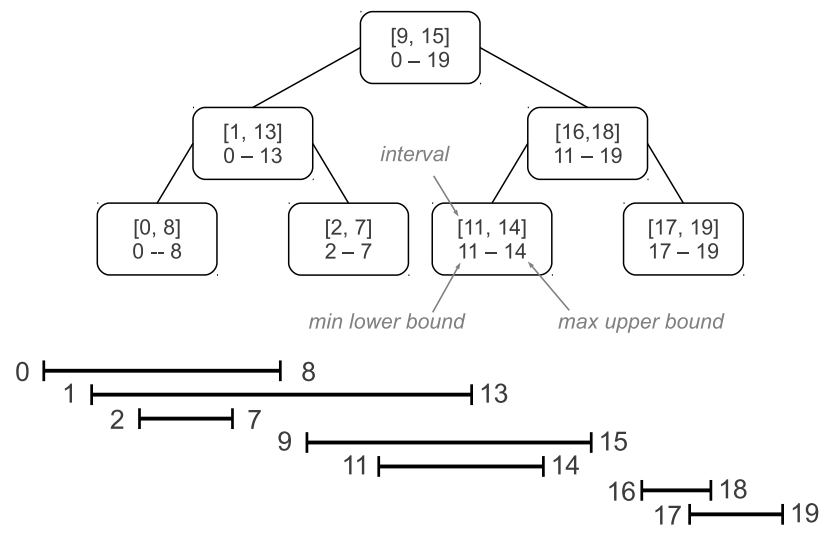

Figure 3. Interval Tree representation of a set of intervals

supports insertions, deletions, and queries to get the list of segments intersecting with a given interval $q$.

Different implementations of the Interval Tree are possible. Priority search trees [13] support insertions and deletions in time $O(\log n)$, and can report all $k$ intersections with a given query interval in time $O(k+\log n)$. For the experimental evaluation described in Section $\nabla$ we implemented the simpler but less efficient variant based on augmented AVL trees [14], described in [15, Chapter 14.3]. We did so in order to trade a slight decrease in asymptotic efficiency for a simpler and more familiar data structure. It should be observed that ITM is not tied to any specific implementation of Interval Tree, therefore any data structure can be used as a drop-in replacement inside the algorithm.

Each node $x$ of the AVL tree holds an interval $x$.in; intervals are sorted according to their lower bounds, and ties are broken by comparing upper bounds. Node $x$ includes two additional fields x.maxupper and $x$.minlower, representing the maximum value of the upper bound and minimum value of the lower bound, respectively, of all intervals stored in the subtree rooted at $x$. We have chosen AVL trees over other balanced search trees, such as red-black trees [16], because AVL trees are more rigidly balanced and therefore allow faster queries.

Figure 3 shows an example of Interval Tree with $n=7$ intervals. Insertions and deletions are handled with the usual rules of AVL trees, with the additional requirement to propagate updates of the maxupper and minlower attributes up to the root. Since the height of an AVL tree is $O(\log n)$, insertions and deletions in the augmented data structure still require $O(\log n)$ time in the worst case. The storage requirement is $O(n)$.

Function InTERVAL-QUeRy $(x, q, \mathbf{M})$, described in Algorithm 4 is used to update matrix $\mathbf{M}$ with all intersections of the update extent $q$ with the segments stored in the subtree rooted at node $x$. The function is invoked as INTERVAL-QUERY(T.root, $q, \mathbf{M})$. The basic idea is very similar to a conventional item lookup in a binary search tree,
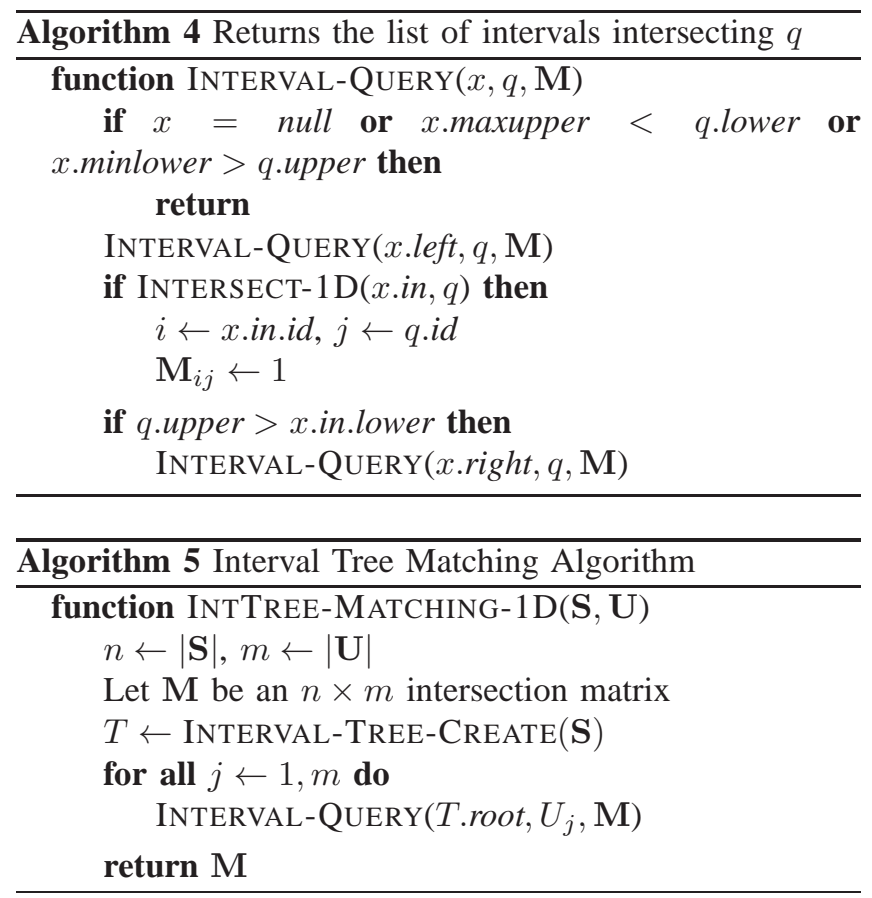

the difference being that at each node $x$ both the $x$.minlower and $x$.maxupper fields are used to drive the exploration of the tree; also, the search might proceed on both the left and right child of node $x$. Algorithm 4 can identify all $k$ intersections between $q$ and all $n$ intervals stored in the tree $T$ in time $O(\min \{n,(k+1) \log n\})$.

The complete ITM matching procedure can now be easily described in Algorithm 5. First, an Interval Tree $T$ is created from the subscription extents in $\mathbf{S}$. Then, for each update extent $U_{j} \in \mathbf{U}$, function INTERVAL-QUERY is invoked to identify all subscriptions that intersect $U_{j}$.

Asymptotic Running Time: If there are $n$ subscription and $m$ update extents, the Interval Tree of subscriptions can be created in time $O(n \log n)$ and requires space $O(n)$; the total query time is $O(\min \{m n,(K+1) \log n\}), K \leq n m$ being the number of intersections involving all subscription and all update intervals. Note that we can assume without loss of generality that $n \leq m$ (if this is not the case, we can switch the role of $\mathbf{S}$ and $\mathbf{U})$.

Parallelizing ITM: Algorithm [5 can be trivially parallelized, since all $m$ queries on $T$ are independent. Note that function INTERVAL-QUERY modifies the intersection matrix $M$ passed as parameter; however, each invocation of INTERVAL-QUERY modifies a different column of $\mathbf{M}$, therefore no conflicts arise. In Section $\mathrm{V}$ we will illustrate the results of experimental investigations on the scalability of the parallel implementation of ITM.

Dynamic interval management: Another interesting feature of ITM is that it can easily cope with dynamic intervals. In most applications, extents can move and grow/shrink dynamically; if an update extent, say $U_{j}$, changes its position 
Table I

DDM ALGORITHMS CONSIDERED IN THE EXPERIMENTAL EVALUATION

\begin{tabular}{lll}
\hline Algorithm & Computational Cost & $\begin{array}{l}\text { Additional } \\
\text { Space }\end{array}$ \\
\hline Brute Force & $O(n m)$ & none \\
Sort-Based & $O((n+m) \log (n+m)+n m)$ & $O(n+m)$ \\
Interval Tree & $O(\min \{m n,(K+1) \log n\})$ & $O(n)$ \\
\hline
\end{tabular}

or size, then it is necessary to recompute column $j$ of matrix M. The brute force approach applied to $U_{j}$ alone gives an $O(n)$ algorithm, since it is only necessary to identify overlaps between $U_{j}$ and all $n$ subscription segments. An extension of SBM capable of updating intersection information efficiently has been proposed [8], with an asymptotic cost that depends on various factors (e.g., upper bound of the dimension, maximum bound shift in a region modification). On the other hand, we can use two Interval Trees $T_{U}$ and $T_{S}$, holding the set of update and subscription extents, respectively, to recompute the intersections efficiently. If an update extent $U_{j}$ is modified, we can identify the subscriptions overlapping $U_{j}$ in time $O(\min \{n,(k+1) \log n\})$ by performing a query on $T_{S}$. Similarly, if a subscription extent $S_{i}$ changes, the list of intersections can be recomputed in time $O(\min \{m,(k+1) \log m\})$ using $T_{U}$. Maintenance of both $T_{U}$ and $T_{S}$ does not affect the asymptotic cost of ITM.

\section{EXPERIMENTAL EVALUATION}

The performance of a DDM service can be influenced by many different factors, including: (i) the computational cost of the DDM matching algorithm; (ii) the memory footprint; (iii) the communication overhead of the parallel/distributed architecture where the simulation is executed and (iv) the cost of sending and discarding irrelevant events at the destination, if any.

The communication overhead depends on the hardware platform over which the simulation model is executed, and also on the implementation details of the communication protocol used by the simulation middleware. Therefore, factor (iii) above is likely to equally affect any DDM algorithm in the same way.

The cost of discarding irrelevant notifications applies only to approximate matching algorithms, such as GB matching, that can report spurious intersections (unless spurious intersections are cleaned up at the sender side). The BF, SBM and ITM algorithms do not suffer from this problem since they never return spurious intersections. Besides, in [7] and [9] the authors show that for relevant cases the SBM algorithm has better performance than GB matching. Therefore, in the performance evaluation study we focused on the exact matching algorithms above, where only factors $(i)$ and (ii) should be considered.

Table I summarizes the computational and memory requirements of the DDM algorithms considered in the exper- imental evaluation. All costs are expressed in term of the number of subscription extents $n$, update extents $m$, and total number of intersections $K$. The "Additional Space" column specifies the additional memory required by each algorithm, excluding the space needed to maintain the lists of intervals, and excluding also the space required by the intersection matrix. As discussed in the previous sections, an extra $O(n m)$ space is required by all algorithms to store the full intersection matrix.

It is important to observe that the asymptotic costs reported in Table I may have little significance when evaluating the actual performance of the algorithms, since in practice the constants hidden in the asymptotic notation may play a major role and should not be ignored. For example, as already explained in Section [II] the weight of the term $\mathrm{nm}$ in the cost of SBM is likely very low since it is originated from simple operations on bit vectors. For these reasons, we performed a set of experimental evaluations whose outcome will be illustrated in this section.

For better comparability of our results with those reported by other research papers, we considered $d=1$ dimensions, and used the methodology and parameters employed in [7]. The first parameter is the total number of extents $N$. We considered a total number of extents in the range $\left[50 \times 10^{3}, 500 \times 10^{3}\right]$. In all cases, $n=N / 2$ are subscription extents and $m=N / 2$ are update extents. All extents are randomly placed on a segment of total length $L=1 \times 10^{6}$. All extents have the same length $l$ that is computed in order to obtain the desired overlapping degree $\alpha$, defined as:

$$
\alpha=\frac{\sum \text { area of extents }}{\text { area of the routing space }}=\frac{N \times l}{L}
$$

Therefore, for a given value of $\alpha$ and $N$, the length $l$ of each segment can be computed as $l=\alpha L / N$. The overlapping degree is an indirect measure of the total number of intersections among subscription and update extents. While the cost of BF and SBM is not affected by the number of intersections, this is not the case for ITM. We considered the same values for $\alpha$ as in [7], namely $\alpha \in\{0.01,1,100\}$.

The experimental evaluation has been performed on an Intel(R) Core(TM) i7-2600 $3.40 \mathrm{GHz}$ CPU with 4 physical cores with Hyper-Threading (HT) technology [17]. The system has 16 GB of RAM and runs Ubuntu 11.04 (x86_64 GNU/Linux, 2.6.38-16-generic \#67-Ubuntu SMP). HT works by duplicating some parts of the processor except the main execution units. From the point of view of the Operating System, each physical processor core corresponds to two logical processors. Many studies from Intel and others have shown that when HT is available, many multithreaded applications can have a performance boost in the range from 16 to $28 \%$ [17. The algorithms have been implemented in $\mathrm{C}$ and compiled with gcc version 4.5.2 using the -03 flag. The SBM algorithm has been implemented according to the improved version described in [7, Section 
4.2], which is more efficient than the basic version shown in Algorithm 3 The parallel versions of BF and ITM have been obtained from the sequential implementation by enabling OpenMP [18] directives in the code.

The performance metric of interest is the total (wall clock) execution time needed to compute the intersection matrix; this time always includes any preprocessing (e.g., the time required by ITM to build the Interval Tree or the time needed by SBM to sort the vector of endpoints). Each measure is the average of 30 independent executions, in order to get statistically valid results. To foster the reproducibility of our experiments, all the source code used in this performance evaluation, and the raw data obtained in the experiments execution, are freely available on the research group website [19] with a Free Software license.

Sequential implementation: We start by comparing the performance of the sequential implementations of BF, SBM and ITM. Figure 4 shows the total execution time of each algorithm as a function of the number of extents $N$, with low, medium and high overlapping degrees $\alpha$.

The vertical scale has been set to allow an easier comparison of SBM and ITM; since Brute Force is the slower algorithm, its execution time goes quickly out of scale. We see that ITM is faster than SBM, but the gap between them tend to close as the overlapping degree $\alpha$ grows. We also observe that the execution time of SBM is unaffected by the value of $\alpha$, which is expected by observing in Table I that its cost does not depend on $K$.

Parallel implementation: We now study the performance of a parallel implementation of ITM. As stated in Section IV ITM can be trivially parallelized using a multithread implementation in which each thread is assigned a subset of the queries. Parallel versions of ITM and BF have been obtained by enabling OpenMP directives in the source code. To the best of our knowledge no parallel versions of SBM have been proposed, and as explained in Section III the sequential step of SBM is affected by a loop-carried dependency which can not be easily avoided.

Figure 5 shows the execution time of the sequential SBM with three configurations of ITM in which a different number of concurrent threads is used. We consider the case in which $\alpha=100$, corresponding the the scenario depicted in Figure 4(c) As expected, the parallel ITM can exploit multiple processor cores to increase the gap from SBM. The practical effect is that ITM remains competitive for larger number of intersections.

To understand the scalability of the parallel version of ITM we compute the speedup $S_{p}$ as a function of the number $p$ of execution threads, where $S_{p}$ is defined as the ratio of the execution time of the sequential implementation and the execution time with $p$ threads.

Figure 6 shows the speedup of parallel BF and parallel ITM. Despite its inefficiency in terms of wall-clock time, $\mathrm{BF}$ has been considered here because it is so easily paral-

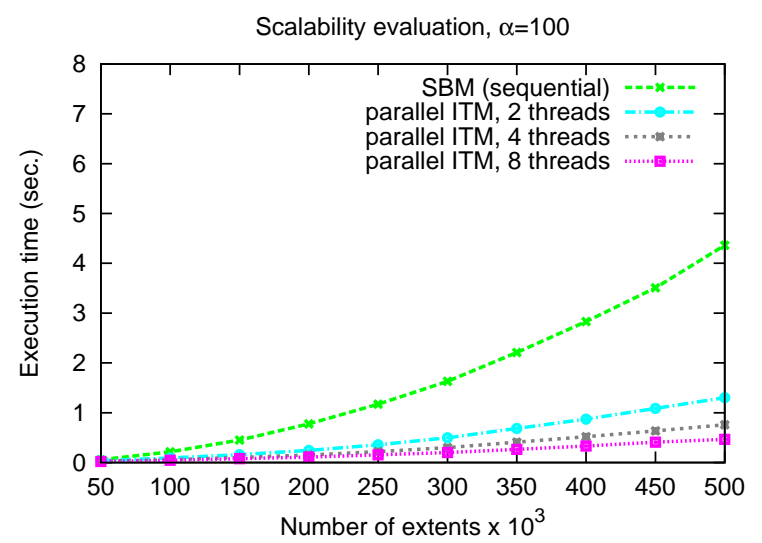

Figure 5. Execution time of SBM (sequential) and ITM (parallel), $\alpha=$ 100 , lower is better

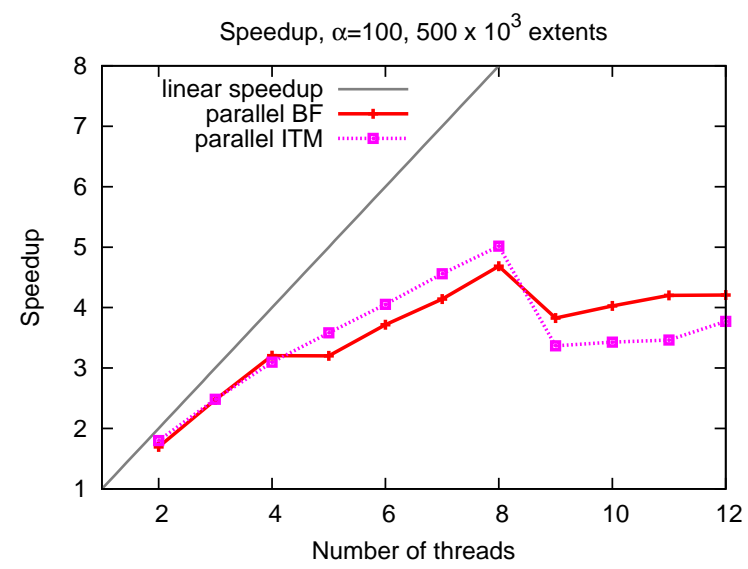

Figure 6. Speedup of parallel $\mathrm{BF}$ and parallel ITM, $N=500 \times 10^{3}$ extents, $\alpha=100$, higher is better

lelizable that it provides baseline values for the speedup. Interestingly, ITM scales better than BF; this is probably due to the improved locality achieved by the Interval Tree, since the intervals stored by the nodes near the root are likely kept in cache. Figure 6 shows also the effect of HT: when the number of threads $p$ is the in the range $1, \ldots, 4$ each execution thread is allocated on a dedicated physical core. When the number of threads exceeds the number of physical cores, then multiple threads are allocated to the same core. As said before, HT does provide a performance boost, but unfortunately not comparable to that provided by an actual physical core with independent execution units. The speedup drops when the number of threads exceeds the number of logical cores.

Finally, Figure 7 summarizes the execution time of parallel ITM over a range of overlapping degrees $(0<\alpha \leq 100)$ and for an increasing number of extents (from $50 \times 10^{3}$ to $500 \times 10^{3}$ ). The plot supports the fact that the running time 


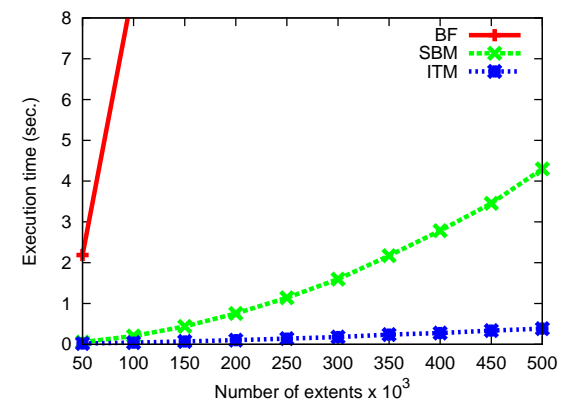

(a) $\alpha=0.01$

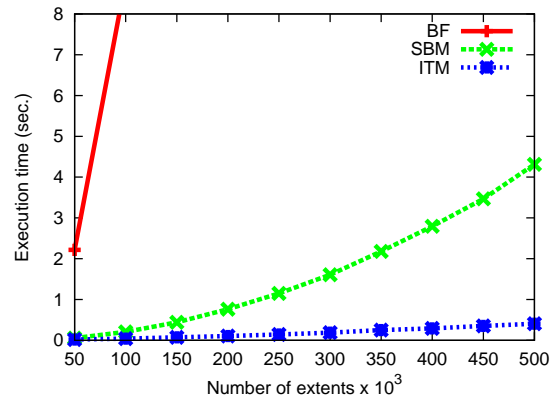

(b) $\alpha=1$

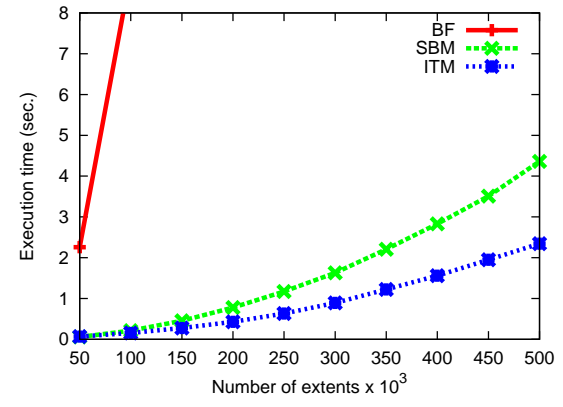

(c) $\alpha=100$

Figure 4. Execution time in seconds as a function of the number $N$ of extents (lower is better). The vertical linear scale, as opposed to logarithmic scale, has been defined to allow easier comparison between SBM and ITM

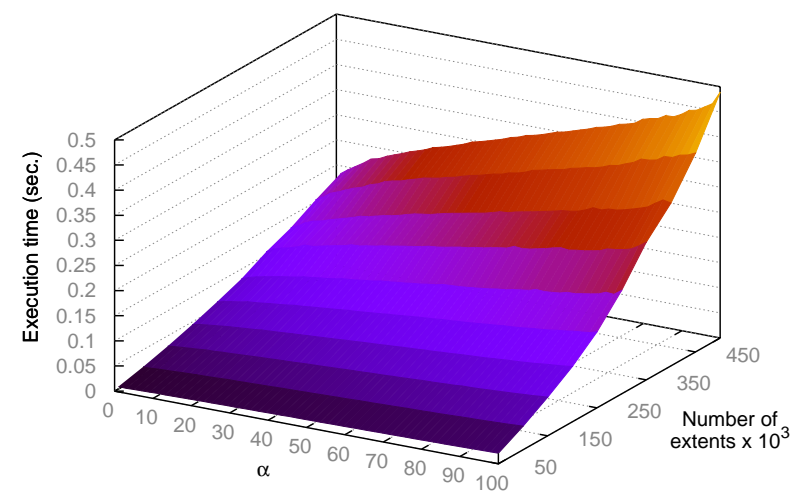

Figure 7. Execution time of parallel ITM, 8 execution threads, lower is better

of ITM depends on both the number of matches $K$ and the input size $N$; in our experiments the value of $K$ is directly correlated with the overlap factor $\alpha$, hence the shape of the graph.

\section{CONCLUSIONS AND FUTURE WORKS}

In this paper we described ITM, a parallel algorithm based on Interval Trees that can be used to solve the $d$-rectangle intersection problem for DDM. ITM uses an augmented AVL tree data structure to store a set of intervals, allowing fast intersection queries. ITM is of practical interest since it can be implemented quite easily; a prototype has been built and is available at [19]. Both the sequential and the parallel implementation of ITM has been evaluated experimentally; the results show that the sequential implementation of ITM compares favorably with Sort-Based Matching, the current best solution to the DDM matching problem. The parallel version of ITM shows good scalability, achieving a $\tilde{5}$ speedup on a four core, hyperthreaded Intel i7 processor.

We are currently extending the ITM prototype as described in Section IV to solve the dynamic DDM matching problem, where extents can be moved or resized dynamically. Furthermore, we are including support for ITM in the GAIA/ARTİS parallel simulation middleware [20]. This will allow us to test ITM in real simulation models, to further assess its performance.

\section{ACKNOWLEDGMENT}

The authors would like to thank Piero Fariselli for kindly providing the server on which the experimental evaluation described in Section $\mathrm{V}$ has been done.

\section{NOTATION}

$\mathbf{S}:=$ Subscription set $\mathbf{S}=\left\{S_{1}, \ldots, S_{n}\right\}$

$\mathbf{U}:=$ Update set $\mathbf{U}=\left\{U_{1}, \ldots, U_{m}\right\}$

$n:=$ Number of subscription extents

$m:=\quad$ Number of update extents

$N$ := Total number of subscription and update extents

$\mathbf{M}:=n \times m$ intersection matrix

$K:=$ Number of intersections, $K \leq n m$

$\alpha:=$ Overlapping degree

\section{REFERENCES}

[1] "IEEE Standard for Modeling and Simulation (M\&S) High Level Architecture (HLA)-Framework and Rules," IEEE Std 1516-2010 (Rev. of IEEE Std 1516-2000), pp. 1-38, 2010.

[2] J. Rosenberg, "Geographical data structures compared: A study of data structures supporting region queries," ComputerAided Design of Integrated Circuits and Systems, IEEE Transactions on, vol. 4, no. 1, pp. 53-67, 1985.

[3] A. Guttman, "R-trees: a dynamic index structure for spatial searching," SIGMOD Rec., vol. 14, no. 2, pp. 47-57, Jun. 1984.

[4] TILERA: TILE-Gx Processor $\quad$ Family, http://www.tilera.com/products/processors/TILE-Gx_Family, 2013.

[5] M. Petty and A. Mukherjee, "Experimental comparison of d-rectangle intersection algorithms applied to HLA data distribution," in Proceedings of the 1997 Distributed Simulation Symposium, 1997, pp. 13-26. 
[6] F. Devai and L. Neumann, "A rectangle-intersection algorithm with limited resource requirements," in Proc. 10th IEEE Int. Conf. on Computer and Information Technology, ser. CIT ' 10. Washington, DC, USA: IEEE Computer Society, 2010, pp. 2335-2340.

[7] C. Raczy, G. Tan, and J. Yu, "A sort-based DDM matching algorithm for HLA," ACM Trans. Model. Comput. Simul., vol. 15, no. 1, pp. 14-38, Jan. 2005.

[8] K. Pan, S. J. Turner, W. Cai, and Z. Li, "A dynamic sort-based DDM matching algorithm for HLA applications," ACM Trans. Model. Comput. Simul., vol. 21, no. 3, pp. 17:1-17:17, Feb. 2011.

[9] J. Ahn, C. Sung, and T. G. Kim, "A binary partitionbased matching algorithm for data distribution management," in Simulation Conference (WSC), Proceedings of the 2011 Winter, 2011, pp. 2723-2734.

[10] R. M. Layer, K. Skadron, G. Robins, I. M. Hall, and A. R. Quinlan, "Binary interval search (BITS): A scalable algorithm for counting interval intersections," Bioinformatics, 2012.

[11] A. Boukerche and C. Dzermajko, "Performance comparison of data distribution management strategies," in Proc. 5th IEEE Int. Workshop on Distributed Simulation and Real-Time Applications, ser. DS-RT '01. Washington, DC, USA: IEEE Computer Society, 2001, pp. 67-.

[12] R. Cole, "Parallel merge sort," SIAM Journal on Computing, vol. 17, no. 4, pp. 770-785, 1988.

[13] E. M. McCreight, "Priority search trees," SIAM J. Comput., vol. 14, no. 2, pp. 257-276, 1985

[14] G. Adelson-Velskii and E. M. Landis, "An Algorithm for the Organization of Information," Doklady Akademii Nauk USSR, vol. 146, no. 2, pp. 263-266, 1962.

[15] T. H. Cormen, C. E. Leiserson, R. L. Rivest, and C. Stein, Introduction to Algorithms (3. ed.). MIT Press, 2009.

[16] L. J. Guibas and R. Sedgewick, "A dichromatic framework for balanced trees," in Foundations of Computer Science, 1978., 19th Annual Symposium on, 1978, pp. 8-21.

[17] D. T. Marr, F. Binns, D. L. Hill, G. Hinton, D. A. Koufaty, A. J. Miller, and M. Upton, "Hyper-Threading Technology Architecture and Microarchitecture," Intel Technology Journal, vol. 6, no. 1, Feb. 2002.

[18] L. Dagum and R. Menon, "OpenMP: An industry-standard [API for shared-memory programming," IEEE Comput. Sci. Eng., vol. 5, pp. 46-55, January 1998.

[19] "Parallel And Distributed Simulation (PADS) research group," http://pads.cs.unibo.it 2013.

[20] L. Bononi, M. Bracuto, G. D’Angelo, and L. Donatiello, "A new adaptive middleware for parallel and distributed simulation of dynamically interacting systems," in Proc. 8th IEEE Int. Symp. on Distributed Simulation and Real-Time Applications. Washington, DC, USA: IEEE Computer Society, 2004, pp. 178-187. 